\title{
Guest Editorial Preface Deep Learning, Ubiquitous, and Toy Computing
}

\begin{abstract}
ABOUT THIS ISSUE
The pervasive nature of digital technologies as witnessed in industry, services, and everyday life has given rise to an emergent, data-focused economy stemming from many aspects of human individual and ubiquitous applications. The richness and vastness of these data are creating unprecedented research opportunities in many fields, including urban studies, geography, economics, finance, entertainment, and social science, as well as physics, biology and genetics, public health, and many other smart devices. In addition to data, text, and machine mining research, businesses and policymakers have seized on deep learning technologies to support their decisions and proper growing smart application needs. As businesses build out emerging hardware and software infrastructure, it becomes increasingly important to anticipate technical and practical challenges and to identify best practices learned through experience in this research area. Deep learning employs software tools from advanced analytics disciplines such as data mining, predictive analytics, text, and machine learning based on a set of algorithms that attempt to model high-level abstractions in data by using multiple processing layers with complex structures or nonlinear transformations. At the same time, the processing and analysis of deep learning applications present methodological and technological challenges. Further, deep learning applications have an advantage by a rise in sensing technologies as witnessed in both the number of sensors and the rich diversity of sensors ranging from cell phones, personal computers, and health tracking appliances to Internet of Things technologies designed to give contextual, semantic data to entities in a ubiquitous environment that previously could not contribute intelligence to key decisions and smart devices. Recently, deep learning technologies have been applied to toy computing. Toy computing is a recently developing concept that transcends the traditional toy into a new area of computer research using ubiquitous technologies. A toy in this context can be effectively considered a computing device or peripheral called Smart Toys. We invite research and industry papers related to these specific challenges and others that are driving innovation in deep learning, ubiquitous, and toy computing.

The goal of this special issue is to present both novel and industrial solutions to challenging technical issues, as well as compelling smart application use cases. This special issue shares related practical experiences to benefit the reader and provides clears proof that deep learning technologies are playing an ever-increasing important and critical role in supporting ubiquitous and toy computing applications-a new cross-discipline research topic in computer science, decision science, and information systems. All eight papers in this issue will have deep research results.

In the first article, "Pegadas: A Portal for Management and Activities Planning With Games and Environments for Education in Health," Machado et al. [2017] focus on developing a Web portal that offers services for organizing and sequencing serious games and virtual environments that allow evaluating the performance of the user. The portal presents the potential of taking advantage of serious games for education and training in health by presenting a comprehensive approach that may help to assist the planning, management, and evaluation of user-oriented health activities. The work also presents a flexible approach that allows integrating different serious games and virtual environments that may have further applications in other fields.
\end{abstract}

2018 Copyright is held by the owner/author(s).

1544-3574/2018/04-ART1

https://doi.org/10.1145/3180663

ACM Computers in Entertainment, Vol. 16, No. 2, Article 1. Publication date: April 2018. 
In the second article, "SimCEC: A Collaborative VR-Based Simulator for Surgical Teamwork Education," Machado et al. [2017] present a collaborative virtual reality simulator for surgical teamwork education focused on basic routines for teams addressing design and implementation challenges associated with real-time feedback, collaborative training, interdisciplinary integration of curricula, and continuous evaluation.

Next, in the third article, "Evolutionary Algorithm for a Better Gaming Experience in Rehabilitation Robotics," Andrade et al. [2017] discuss the use of two evolutionary algorithms to the dynamic difficulty adjustment of a serious game for rehabilitation with robotics. For quality of the game experience, it is of great importance to avoid boredom and frustration of the players, especially for those with severe mobility limitations resulting from stroke, cerebral palsy, and spinal cord injuries. The algorithm changes the difficulty depending on the player's skills and builds upon games played and simulated profiles. The results indicate that the algorithm allows identifying a set of coefficients that allow adjusting the difficulty for players.

Further, in the fourth article, "Using a Social Educational Network to Facilitate Peer-Feedback for a Virtual Simulation," Khan et al. [2017] present a preliminary study that examined the application of a customized social educational network to facilitate medical-based cultural competence training. The study employed questionnaires to gauge both the feasibility of a social educational network to facilitate peer-feedback and the participant's satisfaction with using and interacting with it. The results highlight the potential of the network in supporting learning in a wide variety of disciplines where engaging and entertainment applications are employed to develop skills.

Next, in the fifth article, "A Serious Game for Anesthesia-Based Advanced Cardiovascular Life Support Training," Shegawa et al. [2017] present a serious game for anesthesia-based advanced cardiovascular life support skills training in crisis resource management. The work builds on the viability of virtual reality through a consumer-level head-mounted display to provide trainees the opportunity to interactively react to a simulated medical emergency within a virtual operating room while providing an interactive and engaging training experience. Results examining the usability (the ease of use of the serious game and its interface) of the serious game and its ability to engage trainees indicate that it is interactive, immersive fun, and engaging, showing promise as a complementary training tool.

Then in the sixth article, "Have We Met Before? Using Consumer-Grade Brain-Computer Interfaces to Detect Unaware Facial Recognition,” Bellman et al. [2017] present an experiment using electroencephalographic signals to study the brain's unaware reactions to seeing faces using consumer-grade devices. Results indicate a high accuracy from both the brain-computer interface and out-of-the-box classification methods. Possible applications of this work assist law enforcement agencies or people with disabilities, or in entertainment where brain signals are learned by software to provide additional input methods rather than the standard mouse and keyboard or game-pad interactions.

In the seventh article, "Natural Walking in Virtual Reality: A Review," Nilsson et al. [2017] review the current state of the art of walking in virtual reality by analyzing numerous techniques and challenges. Two main traits are presented, focusing on how to achieve unconstrained walking through repositioning systems, locomotion based on proxy gestures, and redirected walking, in addition to multisensory feedback to increase realism and immersion through external sensory information (visual, auditory, and cutaneous), internal sensory information (vestibular and kinesthetic/proprioceptive), and efferent information.

Last, in the eighth article, "User Testing Without the User: Opportunities and Challenges of an AI-Driven Approach in Games User Research," Stahlke and Mirza-Babaei [2018] discuss the opportunities and challenges in the use of behavioral modeling to create independent user agents 
driven by artificial intelligence (AI) and propose a configurable population of AI players that can provide a data-rich supplement to current approaches in games user research.

Alvaro Joffre Uribe Quevedo

Faculty of Business and Information Technology University of Ontario Institute of Technology, Canada

Email: alvaro.quevedo@uoit.ca

Patrick C. K. Hung

Faculty of Business and Information Technology

University of Ontario Institute of Technology, Canada

Email: patrick.hung@uoit.ca

Shih-Chia Huang

Department of Electronic Engineering

National Taipei University of Technology, Taiwan

Email: schuang@ntut.edu.tw

Sarajane Marques Peres

School of Arts, Sciences and Humanities

University of São Paulo, Brazil

Email: sarajane@usp.br

\section{ABOUT THE GUEST EDITORS}

Alvaro Joffre Uribe Quevedo is an assistant professor at the University of Ontario Institute of Technology (UOIT). He received his bachelor degree in mechatronics engineering from Universidad M. Nueva Granada, Colombia, followed by master and doctoral degrees in mechanical engineering from the State University of Campinas, Brazil. In September 2016, he concluded his postdoctoral appointment at the Games Institute at the University of Waterloo, conducting research on the effects of games in medical training. Prior to joining UOIT, he worked as an assistant professor at Universidad M. Nueva Granada, where he conducted research on exergames, medical simulation, and immersive virtual reality in medical procedures. His research interests are in virtual reality toward the development of applications that take advantage of immersion and interaction while designing and employing emerging technologies and game elements in training and learning scenarios.

Patrick C. K. Hung is a professor at the Faculty of Business and Information Technology, University of Ontario Institute of Technology, Canada. Patrick has been working with Boeing Research and Technology on aviation services-related research with two U.S. patents on the mobile network dynamic workflow system. He is an honorary international chair professor at National Taipei University of Technology in Taiwan, an adjunct professor at Nanjing University of Information Science and Technology in China, and a visiting professor at the University of Sao Paulo in Brazil. In addition, he was an adjunct professor at Wuhan University, a visiting professor at the Shizuoka University and the University of Aizu in Japan, and a guest professor at the University of Innsbruck in Austria, and the University of Trento and the University of Milan in Italy. Before that, he was a research scientist with Commonwealth Scientific and Industrial Research Organization in Australia and additionally worked as a software engineer in the industry in North America. He is a founding committee member of the IEEE International Conference of Web Services (ICWS), the IEEE International Conference on Services Computing (SCC), and the IEEE BigData Congress (BigData Congress). He is also an associate editor of IEEE Transactions on Services Computing and a coordinating editor of Information Systems Frontiers. 
Shih-Chia Huang is a full professor in the Department of Electronic Engineering at National Taipei University of Technology, Taiwan, and an international adjunct professor with the Faculty of Business and Information Technology, University of Ontario Institute of Technology, Canada. He is currently the chair of the IEEE Taipei Section Broadcast Technology Society. Shi-Chia has published more than 50 journal and conference papers and holds more than 40 patents in the United States, Europe, Taiwan, and China. In addition, he is an associate editor of the fournal of Artificial Intelligence and a guest editor of Information Systems Frontiers and the International fournal of Web Services Research. He is also the Applications Track Chair and Program Committee Chair of the IEEE BigData Congress, as well as the IEEE BigData Taipei Satellite Session in 2015, and was Program Committee Chair of the IEEE BigData Taipei Satellite Session in 2014. His research interests include intelligent multimedia systems, image processing and video coding, video surveillance systems, cloud computing and big data analytics, and mobile applications and systems.

Sarajane Marques Peres is an associate professor at the University of Sao Paulo, Brazil. She received her doctoral degree in electric engineering from the University of Campinas in 2006, her master degree in manufacturing engineering from the Federal University of Santa Catarina in 1999, and her bachelor degree in computer science from the State University of Maringá, Brazil, in 1996. She worked as assistant professor at the State University of Western Paraná, Brazil, from 1998 to 2005 and the State University of Maringá, Brazil, from 2005 to 2007. Currently, Sarajane is the tutor for the PET Information Systems USP group, under the Tutorial Education Program of the Ministry of Education, Brazil. Her main research interests are computational intelligence, data mining, machine learning, pattern recognition, and gesture analysis.

\section{REFERENCES}

Christopher Bellman, Miguel Vargas Martin, Shane MacDonald, Ruba Alomari, and Ramiro Liscano. 2017. Have we met before? Using consumer-grade brain-computer interfaces to detect unaware facial recognition. CIE-2016-12-0008.R2.

Zain Khan, David Rojas Gualdron, Bill Kapralos, Lawrence Grierson, and Adam Dubrowski. 2017. Using a social educational network to facilitate peer-feedback for a virtual simulation. CIE-2017-03-0006.R2.

Andrade Kieber, Ricardo Joaquim, Glauco Caurin, and Marcio Crocomo. 2017. Evolutionary algorithm for a better gaming experience in rehabilitation robotics. CIE-2017-04-0007.R2.

Liliane Machado, Thaise Costa, Ronei Moraes, Marco Winckler, and Ana Maria Valença. 2017. Pegadas: A portal for management and activities planning with games and environments for education in health. CIE-2017-08-0013.R1.

Liliane Machado, Paulo Paiva, Ana Maria Valença, Thiago Batista, and Ronei Moraes. 2017. SimCEC: A collaborative VRbased simulator for surgical teamwork education. CIE-2017-06-0009.R1.

Niels Nilsson, Rolf Nordhal, Frank Steinicke, and Stefania Serafin. 2017. Natural walking in virtual reality: A review. CIE2017-04-0008.R1.

Robert Shewaga, Alvaro Uribe Quevedo, Bill Kapralos, Kenneth Lee, and Fahad Alam. 2017. A serious game for anesthesiabased advanced cardiovascular life support training. CIE-2017-02-0004.R1.

Samantha Stahlke and Pejman Mirza-Babaei. 2018. User testing without the user: Opportunities and challenges of an AIdriven approach in games user research. CIE-2018-01-0001. 